\title{
Nanophase-Separated Ionomers for Energy and Environment
}

\author{
Dr. Philippe Knauth \\ Aix Marseille Université, CNRS \\ Marseille, France
}

\begin{abstract}
Ionomers are ion-conducting polymers that have ionophoric groups anchored on the polymer chain. Due to the existence of hydrophobic domains (polymer backbone) and hydrophilic domains (ionic groups), they generally present a nanophase separation. The ions migrate in hydrated nanochannels percolating through the ionomer. These ion-conducting polymers can be used as innovative and high performance separation membranes for various energy and environmental applications, including for example separators for batteries and fuel cells, ultrafiltration and water purification membranes. The presentation will include cation-conducting, anion-conducting and ampholytic polymers.
\end{abstract}

